\title{
Dual-color microscopy of GFP variants in cultured in hippocampal neurons: direct observation of transport vesicle populations involved in polarized protein sorting.
}

\author{
G. Glover*, S. Kaech*, B. Sampo* and G. Banker*
}

Center for Research on Occupational and Environmental Toxicology, Oregon Health and Science University, 3181 SW Sam Jackson Park Rd. Portland, OR 97239

Membrane proteins are transported in vesicles or tubules that carry their cargo from one subcellular site to another. A crucial step in protein targeting is thought to occur during formation of these vesicles; peptide signals within the protein's sequence interact with soluble factors that govern cargo selection into nascent vesicles. The use of the GFP tag has revolutionized imaging of transport vesicles in live cells, and the development of GFP color variants that can be used in combination (such as CFP and YFP) makes it possible to watch two different proteins travel at the same time in the same cell $[1,2]$. We have applied dual-color, CFP,YFP imaging of transport carriers to a key issue in nerve cell biology, and an issue of importance to cell biology in general: characterizing the vesicle populations that deliver proteins to distinct domains on the plasma membrane.

Neurons in culture are particularly well suited for live cell imaging studies of polarized cells. First, they are relatively flat, so capturing movies of transport carriers in axons and dendrites is possible. In addition, vesicles moving back and forth in axons and dendrites essentially travel along a single axis, which makes them easy to follow and analyze. To examine the specific packaging of membrane proteins into transport carriers, we used GFP color variants to tag dendritically and axonally polarized proteins. To determine if two markers were transported together, we imaged cells expressing CFP and YFP pairs, taking sequential $800 \mathrm{msec}$ exposures in the two channels over a time period of 48 seconds and analyzed vesicle movements in such movies using the kymograph function in Metamorph. When displayed as a kymograph, moving organelles are represented as diagonal lines whose slopes correspond to the velocity of movement; stationary structures appear as horizontal lines. As a control, we made two-color movies of cells co-transfected with TfR-CFP and TfR-YFP (Figure 1), which exhibited a high degree of overlap (about 90\%, see Table 1).

Using this approach, we found that representative axonal (NgCAM) and dendritic (TfR, LDLR) markers were present in separate carrier populations, while the two dendritic markers were found in overlapping carrier populations. In addition, the sorting of LDLR was altered upon mutation of critical residues in its sorting signal, shifting LDLR out of TfR-containing dendritic transport vesicles and into an NgCAM-positive vesicle population (Table 1).

References

[1] P. T. Keller et al. Nature Cell Biology 3, (2001) 140.

[2] This work was supported by NIH Grants MH66179 and NS17112. We thank B. Smoody for preparing hippocampal cultures and J. Luisi Harp for assistance with molecular biology. 


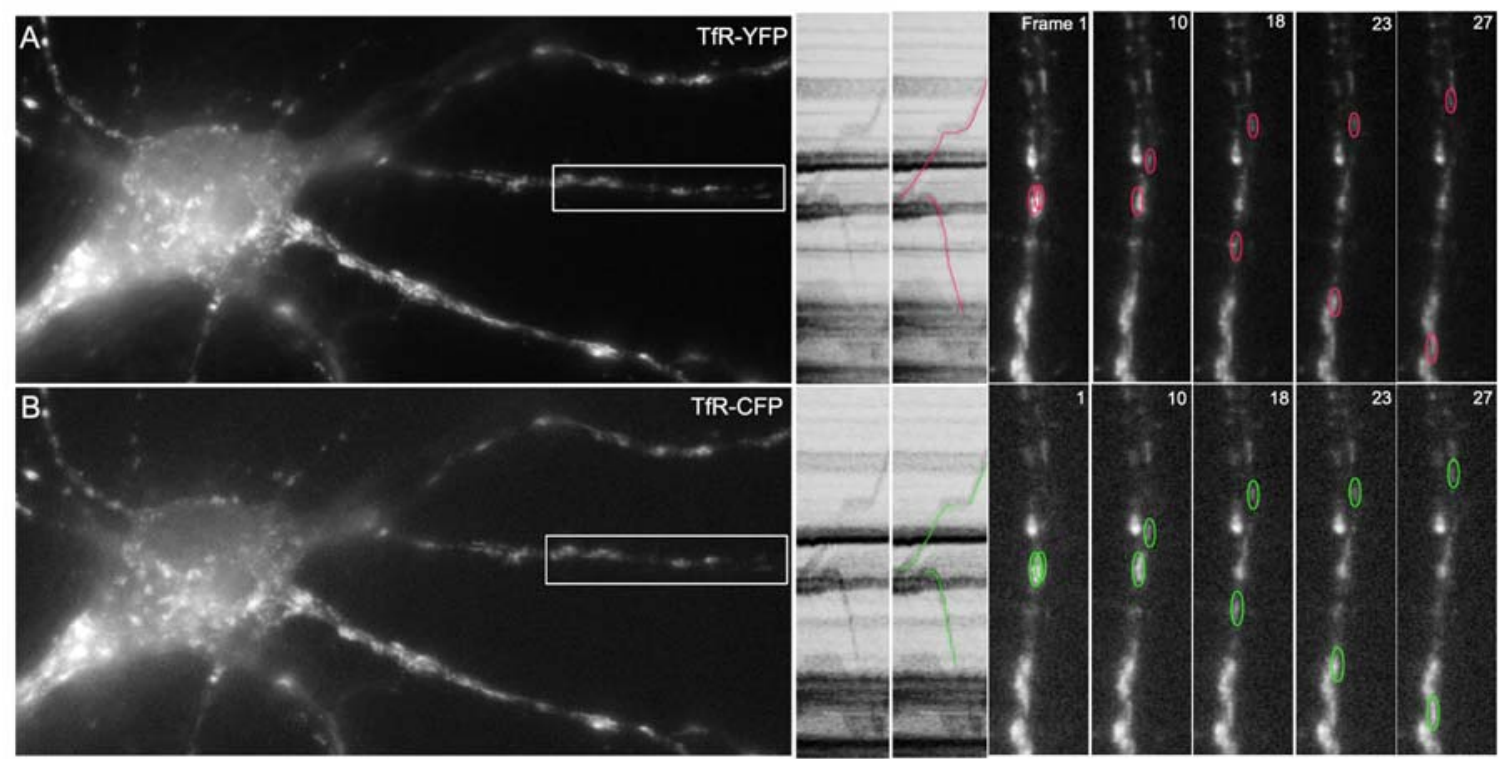

Figure 1. Two-color live cell imaging of transport intermediates containing CFP- and YFP-tagged proteins. A single movie frame from each color shows the overall similarity in the distribution of TfR-YFP and TfR-CFP (A, B, left panel). Kymographs taken from the boxed region are shown in duplicate in the middle panels. Kymographs show the maximal fluorescence intensity across the width of the neurite at each position along it (represented on the vertical axis) for each successive time point (shown along the horizontal axis). Diagonal lines represent moving carriers, with a positive slope corresponding to movement away from the cell body; horizontal lines represent stationary structures. Individual movie frames are shown at right. Scale bar $5 \mu \mathrm{m}$.

Table 1 Quantification of overlap between YFP/CFP pairs in moving carriers

\begin{tabular}{|c|c|c|c|c|}
\hline \multicolumn{2}{|c|}{ marker pairs } & \multirow{2}{*}{$\begin{array}{l}\text { \%YFP overlapping } \\
\text { with } \mathrm{CFP}^{1}\end{array}$} & \multirow{2}{*}{$\begin{array}{c}\text { \%CFP overlapping } \\
\text { with } \mathrm{YFP}^{1}\end{array}$} & \multirow{2}{*}{$\begin{array}{c}\text { \# transport events / \# } \\
\text { cells }\end{array}$} \\
\hline YFP & CFP & & & \\
\hline TfR & TfR & $93 \pm 12 \%$ & $89 \pm 9 \%$ & $109 / 9$ \\
\hline LDLR & $\mathrm{TfR}$ & $66 \pm 13 \%$ & $74 \pm 13 \%$ & $182 / 8$ \\
\hline LDLRmut & TfR & $12 \pm 17 \% *$ & $8 \pm 12 \% *$ & $281 / 9$ \\
\hline LDLR & NgCAM & $12 \pm 9 \% *$ & $10 \pm 12 \% *$ & $278 / 7$ \\
\hline LDLRmut & NgCAM & $65 \pm 13 \% * *$ & $54 \pm 12 \% * *$ & $338 / 14$ \\
\hline
\end{tabular}

${ }^{1}$ values are mean \pm standard deviation $*$ significantly different from LDLR/TfR; ** significantly different from LDLR/NgCAM 